\title{
Reclassification of Donghaeana dokdonensis Yoon et al. 2006 as Persicivirga dokdonensis comb. nov. and emended descriptions of the genus Persicivirga and of Persicivirga xylanidelens O'Sullivan et al. 2006 \\ Correspondence \\ Olga I. Nedashkovskaya \\ olganedashkovska@piboc.dvo.ru \\ Olga I. Nedashkovskaya, ${ }^{1,2}$ Kae Kyoung Kwon ${ }^{1}$ and Sang-Jin Kim ${ }^{1}$

\author{
${ }^{1}$ Marine Biotechnology Research Center, Korea Ocean Research and Development Institute, \\ PO Box 29, Ansan 425-600, Republic of Korea \\ ${ }^{2}$ Pacific Institute of Bioorganic Chemistry of the Far-Eastern Branch of the Russian Academy of \\ Sciences, Pr. 100 Let Vladivostoku 159, 690022 Vladivostok, Russia
}

The genus Persicivirga was proposed to accommodate Gram-negative, strictly aerobic, heterotrophic, peachorange-pigmented, oxidase- and catalase-negative marine bacteria that belong to the family Flavobacteriaceae, phylum 'Bacteroidetes' (O'Sullivan et al., 2006). The genus comprises a single species, Persicivirga xylanidelens; the type and only strain, designated SW $256^{\mathrm{T}}$, was isolated from a coastal seawater sample in the North Sea (O'Sullivan et al., 2006). At about the same time, another genus and species, Donghaeana dokdonensis, was proposed to accommodate strain DSW $-6^{\mathrm{T}}$, isolated from surface water of the East Sea (Yoon et al., 2006). Shortly after the genera Persicivirga and Donghaeana were described, their very close phylogenetic relationship became evident.

In the present work, the precise taxonomic positions of strains SW $256^{\mathrm{T}}$ and DSW $-6^{\mathrm{T}}$ were investigated by using a polyphasic approach. The results of phylogenetic, genomic and phenotypic analyses supported the affiliation of the two strains to the same genus. The name Persicivirga has priority over the name Donghaeana due to the earlier publication of its description (Rule 24b of Principle 6; Lapage et al., 1992). Hence, it is proposed that strain DSW$6^{\mathrm{T}}$ be transferred to the genus Persicivirga as a distinct species.

A phylogenetic analysis based on published 16S rRNA gene sequences was conducted according to the procedure described previously (Nedashkovskaya et al., 2007). An unambiguously aligned $1211 \mathrm{bp}$ sequence fragment was compared between members of closely related genera retrieved from GenBank. P. xylanidelens SW $256^{\mathrm{T}}$ was the closest relative of $D$. dokdonensis DSW- ${ }^{\mathrm{T}}$, with sequence similarity of $98.2 \%$. This value supports the allocation of strain DSW $-6^{\mathrm{T}}$ to the genus Persicivirga (O'Sullivan et al., 2006). 16S rRNA gene sequence similarities between strain DSW $-6^{\mathrm{T}}$ and its other neighbours, members of the genera Nonlabens, Sandarakinotalea and Stenothermobacter, ranged from 93.1 to $94.3 \%$. The phylogenetic analysis revealed that strain DSW $-6^{\mathrm{T}}$ forms a cluster with the representatives of these genera, but forms a distinct phyletic line with strain SW256 ${ }^{\mathrm{T}}$ that is supported by a high bootstrap value (Fig. 1). In addition, Fig. 1 revealed that the genera Nonlabens and Sandarakinotalea also display high $16 \mathrm{~S}$ rRNA gene sequence similarity $(99.8 \%)$ and should be reclassified (Fig. 1).

The DNA of the two strains was isolated following the method of Marmur (1961) and the DNA G + C content was determined by the thermal-denaturation method (Marmur \& Doty, 1962). The DNA G+C contents of D. dokdonensis DSW- $6^{\mathrm{T}}$ and P. xylanidelens SW $256^{\mathrm{T}}$ were 35.9 and $35.5 \mathrm{~mol} \%$, respectively. These values are close to those obtained by using the HPLC technique (O'Sullivan et al., 2006; Yoon et al., 2006).

The DNA-DNA relatedness between the two strains was determined spectrophotometrically and initial renaturation rates were recorded as described by De Ley et al. (1970). The level of DNA-DNA relatedness between strains DSW $-6^{\mathrm{T}}$ and SW $256^{\mathrm{T}}$ was $33 \%$, a value that justifies the allocation of the two strains to two different species in the genus Persicivirga according to the proposal of Wayne et al. (1987). 


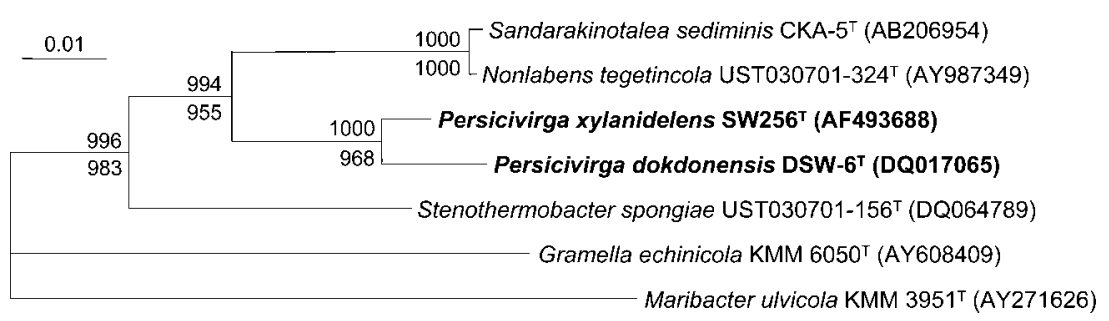

Fig. 1. Rooted neighbour-joining tree based on $16 \mathrm{~S}$ rRNA gene sequences showing the relationship between Persicivirga dokdonensis DSW $-6^{\top}$ and closely related genera of the family Flavobacteriaceae. Bootstrap values $>50 \%$ of 1000 replications are shown at nodes (upper value, Jukes-Cantor/neighbour joining; lower value, maximum likelihood/parsimony). Maribacter ulvicola $\mathrm{KMM} 3951^{\top}$ (AY271626) was used as an outgroup. Bar, 0.01 substitutions per nucleotide position.
Physiological and biochemical features of strains DSW $-6^{\mathrm{T}}$ and SW256 ${ }^{\mathrm{T}}$ were tested as described previously (Nedashkovskaya et al., 2004) and by using API 20E, API 20NE and API ZYM galleries (bioMérieux) according to the manufacturer's instructions, except that the incubation temperature was $28{ }^{\circ} \mathrm{C}$.

Strains DSW-6 ${ }^{\mathrm{T}}$ and SW256 ${ }^{\mathrm{T}}$ share many phenotypic features. They are aerobic, non-gliding bacteria that possess alkaline phosphatase, caseinase and DNase activities. In contrast to previously reported data (O'Sullivan et al., 2006), this study revealed that strain SW $256^{\mathrm{T}}$ is able to produce cytochrome oxidase and catalase and to hydrolyse aesculin, in line with strain DSW- $6^{\mathrm{T}}$. However, the two strains differ from each other by several phenotypic characteristics such as hydrolysis of gelatin, starch and Tween 80, utilization of mannitol and production of several enzymes (Table 1).

Therefore, the phylogenetic evidence and similarity in fatty acid composition (Table 2) and phenotypic features (Table 1) provide a sound basis for reclassification of $D$. dokdonensis in the genus Persicivirga. Differences in several phenotypic characteristics and a low level of DNA-DNA relatedness between the two strains justify the classification of strain DSW- $6^{\mathrm{T}}$ as Persicivirga dokdonensis comb. nov. Emended descriptions of the genus Persicivirga and of $P$. xylanidelens are also proposed to include new data obtained in this study. Table 3 lists the phenotypic properties distinguishing members of the genus Persicivirga from their close relatives.

\section{Emended description of the genus Persicivirga O'Sullivan et al. 2006}

Persicivirga (Per.si.ci.vir'ga. L. neut. n. persicum peach; L. fem. n. virga rod; N.L. fem. n. Persicivirga a peach-coloured rod).

Cells are rod-shaped, non-motile, strictly aerobic and Gram-negative. Chemo-organotrophic. Endospores are not formed. Some strains can produce flexirubin-type pigments. Cytochrome oxidase-, catalase- and alkaline phosphatase-positive. $\mathrm{Na}^{+}$ions are required for growth. The predominant cellular fatty acids are anteiso- $\mathrm{C}_{15: 0}$, iso$\mathrm{C}_{15: 0}$, iso- $\mathrm{C}_{17: 0} 3-\mathrm{OH}$, iso- $\mathrm{C}_{15: 0} 3-\mathrm{OH}, \mathrm{C}_{15: 0}$ and summed feature 3 (consisting of iso- $\mathrm{C}_{15: 0} 2-\mathrm{OH}$ and/or $\mathrm{C}_{16: 1} \omega 7 c$ ). The major isoprenoid quinone is MK-6. As determined by $16 \mathrm{~S}$ rRNA gene sequence analysis, the genus Persicivirga is a member of the family Flavobacteriaceae, phylum 'Bacteroidetes'. The type species is Persicivirga xylanidelens.

Table 1. Differential characteristics of Persicivirga species

Species: 1 , Persicivirga xylanidelens SW256 ${ }^{\mathrm{T}} ; 2$, Persicivirga dokdonensis DSW $-6^{\mathrm{T}}$. Both strains were positive for the following characteristics: respiratory metabolism, presence of esterase (C4), esterase lipase (C8), leucine and valine arylamidase, acid and alkaline phosphatase, naphthol-AS-BI-phosphohydrolase, oxidase and catalase activities and hydrolysis of aesculin, casein, DNA and Tweens 20 and 40. All strains were negative for the following characteristics: gliding motility, presence of cystine arylamidase, $\alpha$-chymotrypsin, $\alpha$ - and $\beta$ galactosidase, $\beta$-glucuronidase, $\alpha$-glucosidase, $N$-acetyl- $\beta$-glucosaminidase, $\alpha$-mannosidase and $\alpha$-fucosidase activities, hydrolysis of agar, cellulose (CM-cellulose and filter paper), urea and chitin, acid production from carbohydrates, utilization of arabinose, glucose, lactose, mannose, sucrose, inositol, sorbitol, adipate, citrate, caprate, gluconate, malate and phenylacetate, nitrate reduction and production of indole and acetoin (Voges-Proskauer reaction). Data are from O'Sullivan et al. (2006), Yoon et al. (2006) and this study.

\begin{tabular}{|lcc|}
\hline Characteristic & $\mathbf{1}$ & $\mathbf{2}$ \\
\hline Flexirubin-type pigments & + & - \\
H $\mathrm{H}_{2}$ S production & + & - \\
Range of: & & \\
$\quad$ Temperatures for growth $\left({ }^{\circ} \mathrm{C}\right)$ & $4-28$ & $4-32$ \\
$\quad$ Salinities for growth $(\% \mathrm{NaCl})$ & $2-6$ & $1-8$ \\
Hydrolysis of: & & \\
$\quad$ Gelatin & - & + \\
$\quad$ Starch & + & - \\
$\quad$ Tween 80 & - & + \\
Utilization of mannitol & + & - \\
Production of lipase $(\mathrm{C} 14)$, trypsin and & & \\
$\quad \beta$-glucosidase & & \\
DNA G $+\mathrm{C}$ content $(\mathrm{mol} \%)$ as determined by: & 34.7 & 36.9 \\
$\quad$ HPLC & 35.5 & 35.9 \\
$\quad$ Thermal denaturation & & \\
\hline
\end{tabular}


Table 2. Cellular fatty acid content (\%) of Persicivirga species

Species: 1 , Persicivirga xylanidelens $\mathrm{SW} 256^{\mathrm{T}} ; 2$, Persicivirga dokdonensis DSW $-6^{\mathrm{T}}$. Fatty acids amounting to $<1 \%$ of the total fatty acids in the two strains are not listed. Both strains were grown for 3 days on marine agar 2216. Strain SW $256^{\mathrm{T}}$ was grown at $20{ }^{\circ} \mathrm{C}$, whereas strain DSW $-6^{\mathrm{T}}$ was grown at $25{ }^{\circ} \mathrm{C}$. $\mathrm{Tr}$, trace $(<1 \%) ;-$, not detected. Data are from O'Sullivan et al. (2006) and Yoon et al. (2006).

\begin{tabular}{|c|c|c|}
\hline Fatty acid & 1 & 2 \\
\hline iso- $\mathrm{C}_{13: 1}$ & 1.9 & - \\
\hline iso- $\mathrm{C}_{14: 0}$ & 3.2 & - \\
\hline anteiso- $\mathrm{C}_{14: 0}$ & 2.6 & - \\
\hline iso- $\mathrm{C}_{15: 0}$ & 18.1 & 19.2 \\
\hline anteiso- $\mathrm{C}_{15: 0}$ & 21.6 & 11.1 \\
\hline $\mathrm{C}_{15: 0}$ & 5.5 & 10.2 \\
\hline $\mathrm{C}_{15: 1}$ & 1.4 & 3.7 \\
\hline iso- $\mathrm{C}_{16: 1}$ & 1.4 & 1.3 \\
\hline iso- $\mathrm{C}_{16: 0}$ & 3.3 & 1.5 \\
\hline $\mathrm{C}_{16: 0}$ & $\operatorname{Tr}$ & 1.5 \\
\hline iso- $\mathrm{C}_{15: 0} 3-\mathrm{OH}$ & 6.9 & 6.7 \\
\hline anteiso- $\mathrm{C}_{15: 0} 3-\mathrm{OH}$ & 2.2 & - \\
\hline $\mathrm{C}_{15: 0} 3-\mathrm{OH}$ & - & 3.3 \\
\hline $\mathrm{C}_{15: 0} 2-\mathrm{OH}$ & - & 1.8 \\
\hline iso- $\mathrm{C}_{17: 1} \omega 9 c$ & 8.5 & 3.5 \\
\hline $\mathrm{C}_{17: 1} \omega 6 c$ & - & 2.9 \\
\hline iso- $\mathrm{C}_{16: 0} 3-\mathrm{OH}$ & 4.7 & 3.5 \\
\hline $\mathrm{C}_{16: 0} 2-0 \mathrm{H}$ & - & 1.5 \\
\hline iso- $\mathrm{C}_{17: 0} 3-\mathrm{OH}$ & 7.1 & 10.5 \\
\hline anteiso- $\mathrm{C}_{17: 0} 3-\mathrm{OH}$ & 4.9 & - \\
\hline $\mathrm{C}_{17: 0} 2-\mathrm{OH}$ & - & 3.0 \\
\hline $\mathrm{C}_{18: 0} \omega 5 c$ & - & 1.7 \\
\hline $\mathrm{C}_{18: 0}$ & - & 2.0 \\
\hline Summed feature $3^{\star}$ & 5.3 & 7.1 \\
\hline
\end{tabular}

${ }^{\star}$ Summed feature 3 consisted of iso- $\mathrm{C}_{15: 0} 2-\mathrm{OH}$ and/or $\mathrm{C}_{16: 1} \omega 7 c$ that could not be separated by GLC using the MIDI system.

\section{Emended description of Persicivirga xylanidelens O'Sullivan et al. 2006}

Persicivirga xylanidelens (xy.la.ni.del'ens. N.L. n. xylanum xylan; L. part. adj. delens destroying; N.L. part. adj. xylanidelens xylan-destroying).

Displays the following properties in addition to those listed in the emended description of the genus and those given by O'Sullivan et al. (2006). Growth occurs with 2-6\% NaCl. Decomposes aesculin, casein, DNA and Tweens 20 and 40. Does not hydrolyse agar or chitin. Acid is not produced from L-arabinose, cellobiose, D-fructose, D-galactose, Dglucose, D-lactose, maltose, D-mannose, melibiose, raffinose, L-rhamnose, ribose, sucrose, trehalose, D-xylose, $\mathrm{N}$ acetylglucosamine, citrate, glycerol or mannitol. LArabinose, D-glucose, D-lactose, D-mannose, sucrose, inositol, mannitol, sorbitol and citrate are not utilized. According to the API ZYM gallery, esterase (C4), esterase lipase (C8), lipase (C14), leucine and valine arylamidase, trypsin, acid phosphatase, $\beta$-glucosidase and naphthol-AS-
Table 3. Differential characteristics of members of the genus Persicivirga and related genera of the family Flavobacteriaceae

Genera: 1, Persicivirga (two species); 2, Nonlabens (one species); 3, Sandarakinotalea (one species); 4, Stenothermobacter (one species). All strains were positive for the following characteristics: respiratory metabolism and presence of catalase, oxidase and alkaline phosphatase activities. All strains were negative for the following characteristics: nitrate reduction, hydrolysis of agar, cellulose (CM-cellulose and filter paper), urea and chitin, acid production from carbohydrates, utilization of lactose and production of indole. +, Positive; -, negative; ND, no data available. Data are from Khan et al. (2006), Lau et al. (2005, 2006), O'Sullivan et al. (2006), Yoon et al. (2006) and this study.

\begin{tabular}{|lcccc|}
\hline Characteristic & $\mathbf{1}$ & $\mathbf{2}$ & $\mathbf{3}$ & $\mathbf{4}$ \\
\hline Gliding motility & - & - & - & + \\
Acetoin production & - & + & $\mathrm{ND}$ & + \\
Range of: & & & & \\
$\quad$ Temperatures for growth $\left({ }^{\circ} \mathrm{C}\right)$ & $4-32$ & $12-44$ & $10-40$ & $20-36$ \\
Salinities for growth $(\% \mathrm{NaCl})$ & $1-8$ & $2-4$ & $0.3-2.5$ & $2-6$ \\
Hydrolysis of: & & & & \\
Aesculin & + & - & - & - \\
Casein & + & - & $\mathrm{ND}$ & - \\
DNA & + & + & + & - \\
Tween 40 & + & + & - & + \\
Acid production from carbohy- & - & - & + & - \\
drates & & & & \\
Utilization of: & & & & \\
D-Arabinose & - & - & - & + \\
D-Glucose & - & + & - & + \\
D-Lactose & - & + & - & \\
Sucrose & - & + & - & + \\
Sorbitol & - & + & - & + \\
Production of: & & & & \\
$\alpha$-Chymotrypsin, cystine aryl- & - & + & $\mathrm{ND}$ & + \\
amidase & & & & \\
$\beta$-Galactosidase, $\alpha$-glucosidase & - & - & $\mathrm{ND}$ & + \\
DNA G+C content (mol\%) & $34-37$ & 33.6 & $35-37$ & 41.0 \\
& & & & \\
\hline
\end{tabular}

BI-phosphohydrolase activities are present, but cystine arylamidase, $\alpha$-chymotrypsin, $\alpha$ - and $\beta$-galactosidase, $\beta$ glucuronidase, $\alpha$-glucosidase, $N$-acetyl- $\beta$-glucosaminidase, $\alpha$-mannosidase and $\alpha$-fucosidase activities are absent. $\mathrm{H}_{2} \mathrm{~S}$ is produced. The predominant fatty acids are anteiso- $\mathrm{C}_{15: 0}$, iso- $\mathrm{C}_{15: 0}$, iso- $\mathrm{C}_{17: 1} \omega 9 c$, iso- $\mathrm{C}_{17: 0} 3-\mathrm{OH}$, iso- $\mathrm{C}_{15: 0} 3-\mathrm{OH}$, $\mathrm{C}_{15: 0}$, summed feature 3 (consisting of iso- $\mathrm{C}_{15: 0} 2-\mathrm{OH}$ and/ or $\mathrm{C}_{16: 1} \omega 7 c$ ), anteiso- $\mathrm{C}_{17: 0} 3-\mathrm{OH}$ and iso- $\mathrm{C}_{16: 0} 3-\mathrm{OH}$.

The type strain, SW $256^{\mathrm{T}} \quad\left(=\mathrm{NCIMB} \quad 14027^{\mathrm{T}}=\mathrm{DSM}\right.$ $16809^{\mathrm{T}}$ ), was isolated from coastal North Sea water, Hope Cove, near Plymouth, UK.

\section{Description of Persicivirga dokdonensis comb. nov.}

Persicivirga dokdonensis (dok.do.nen'sis. N.L. fem. adj. dokdonensis of Dokdo, an island in the East Sea where the type strain was isolated). 
Basonym: Donghaeana dokdonensis Yoon et al. 2006.

Displays the following properties in addition to those listed in the emended description of the genus and those given by Yoon et al. (2006). Mannitol is utilized, but L-arabinose, Dlactose, D-mannose, adipate, caprate, citrate, gluconate, malate, phenylacetate, inositol and sorbitol are not. DNA is hydrolysed.

The type strain, DSW $-6^{\mathrm{T}}\left(=\mathrm{DSM} 17205^{\mathrm{T}}=\mathrm{KCTC} 12402^{\mathrm{T}}\right)$, was isolated from sea water of the East Sea, also known as the Sea of Japan.

\section{Acknowledgements}

We are most grateful to Professor Dr Erko Stackebrandt and Dr Brian J. Tindall from the Deutsche Sammlung von Mikroorganismen und Zellkulturen GmbH, Braunschweig, Germany, for providing us with P. xylanidelens DSM $16809^{\mathrm{T}}$ and to Dr Jung-Sook Lee from the Korean Collection for Type Cultures, Daejon, Republic of Korea, for providing us with $D$. dokdonensis KCTC $12402^{\mathrm{T}}$. This research was supported by grants from the Russian Foundation for Basic Research (no. 08-04-00099), from the Presidium of the Russian Academy of Sciences 'Molecular and Cell Biology' and from the 'Marine and Extreme Genome Research Center Program' supported by the Ministry of Land, Transport and Maritime Affairs of Korea.

\section{References}

De Ley, J., Cattoir, H. \& Reynaerts, A. (1970). The quantitative measurement of DNA hybridization from renaturation rates. Eur J Biochem 12, 133-142.

Khan, S. T., Nakagawa, Y. \& Harayama, S. (2006). Sandarakinotalea sediminis gen. nov., sp. nov., a novel member of the family Flavobacteriaceae. Int J Syst Evol Microbiol 56, 959-963.

Lapage, S. P., Sneath, P. H. A., Lessel, E. F., Skerman, V. B. D., Seeliger, H. P. R. \& Clark, W. A. (1992). International Code of Nomenclature of Bacteria (1990 Revision). Washington, DC: American Society for Microbiology.

Lau, S. C. K., Tsoi, M. M. Y., Li, X., Plakhotnikova, I., Dobretsov, S., Wong, P. K., Pawlik, J. R. \& Qian, P. Y. (2005). Nonlabens tegetincola gen. nov., sp. nov., a novel member of the family Flavobacteriaceae isolated from a microbial mat in a subtropical estuary. Int J Syst Evol Microbiol 55, 2279-2283.

Lau, S. C. K., Tsoi, M. M. Y., Li, X., Plakhotnikova, I., Dobretsov, S., Wu, M., Wong, P. K., Pawlik, J. R. \& Qian, P. Y. (2006). Stenothermobacter spongiae gen. nov., sp. nov., a novel member of the family Flavobacteriaceae isolated from a marine sponge in the Bahamas, and emended description of Nonlabens tegetincola. Int J Syst Evol Microbiol 56, 181-185.

Marmur, J. (1961). A procedure for the isolation of deoxyribonucleic acid from microorganisms. J Mol Biol 3, 208-218.

Marmur, J. \& Doty, P. (1962). Determination of the base composition of deoxyribonucleic acid from its thermal denaturation temperature. J Mol Biol 5, 109-118.

Nedashkovskaya, O. I., Kim, S. B., Han, S. K., Lysenko, A. M., Rohde, M., Rhee, M.-S., Frolova, G. M., Falsen, E., Mikhailov, V. V. \& Bae, K. S. (2004). Maribacter gen. nov., a new member of the family Flavobacteriaceae, isolated from marine habitats, containing the species Maribacter sedimenticola sp. nov., Maribacter aquivivus sp. nov., Maribacter orientalis sp. nov. and Maribacter ulvicola sp. nov. Int J Syst Evol Microbiol 54, 1017-1023.

Nedashkovskaya, O.I., Kim, S.B., Kwon, K.K., Shin, D.S., Xuseong, L., Kim, S.-J. \& Mikhailov, V.V. (2007). Proposal of Algoriphagus vanfongensis sp. nov., transfer of members of the genera Hongiella Yi and Chun 2004 emend. Nedashkovskaya et al. 2004 and Chimaereicella Tiago et al. 2006 to the genus Algoriphagus, and emended description of the genus Algoriphagus Bowman et al. 2003 emend. Nedashkovskaya et al. 2004. Int J Syst Evol Microbiol 57, 19881994.

O’Sullivan, L. A., Rinna, J., Humphreys, G., Wightman, A. J. \& Fry, J. C. (2006). Culturable phylogenetic diversity of the phylum 'Bacteroidetes' from river epilithon and coastal water and description of novel members of the family Flavobacteriaceae: Epilithonimonas tenax gen. nov., sp. nov. and Persicivirga xylanidelens gen. nov., sp. nov. Int J Syst Evol Microbiol 56, 169-180.

Wayne, L. G., Brenner, D. J., Colwell, R. R., Grimont, P. A. D., Kandler, P., Krichevsky, M. I., Moore, L. H., Murray, R. G. E., Stackebrandt, E. \& other authors (1987). Report of the ad hoc committee on reconciliation of approaches to bacterial systematics. Int J Syst Bacteriol 37, 463-464.

Yoon, J. H., Kang, S. J., Lee, C. H. \& Oh, T. K. (2006). Donghaeana dokdonensis gen. nov., sp. nov., isolated from sea water. Int J Syst Evol Microbiol 56, 187-191. 